Pseudoexon activation in nicotinamide nucleotide transhydrogenase (NNT) in two siblings with Familial Glucocorticoid Deficiency

${ }^{1}$ Centre for Endocrinology, William Harvey Research Institute, John Vane Science Centre, Queen Mary, University of London, Charterhouse Square, London, EC1M 6BQ, UK, ${ }^{2}$ Department of Endocrinology Princess Margaret Hospital, Child and Adolescent Services \& School of Pediatrics and Child Health, University of Western Australia Perth Australia, ${ }^{3}$ Department of Diagnostic Genomics, PathWest Laboratory Medicine, Nedlands, Western Australia 6009

\title{
Introduction
}

Intronic DNA frequently encodes potential exonic sequences called pseudoexons, i.e. sequences very similar to exons with $5^{\prime}$ and $3^{\prime}$ splice-sites that are ignored by the cellular splicing machinery and therefore not included into mature mRNA. To date aberrant pseudoexon inclusion has been implicated in approximately 50 genes in various diseases.

\section{Clinical presentation}

The proband (Fig. 1A, II:2) presented at 21 months of age, unresponsive with hypoglycaemia (serum blood glucose 0.6 mmol/L (normal range (N.R) 3.0-5.4 mmol/L). Endocrine evaluation subsequent to resuscitation indicated adrenal insufficiency with elevated plasma ACTH $492 \mathrm{pmol} / \mathrm{L}$ (N.R 2.2-13.3 pmol/L), low cortisol $30 \mathrm{nmol} / \mathrm{L}$ (N.R. 138-635 $\mathrm{nmol} / \mathrm{L}$ ), normal serum aldosterone (192 pmol/L) and appropriately suppressed insulin <2mU/L with ketosis. The child had hyperpigmentation of the skin (Fig. 1B). Hydrocortisone therapy was commenced upon diagnosis of FGD. A sibling (Fig. 1A, II:5), 4 years younger than the proband had a short synacthen test (SST) performed on day 4 of life: baseline cortisol $38 \mathrm{nmol} / \mathrm{L}$ with a 60 minute peak of $380 \mathrm{nmol} / \mathrm{L}$. Hydrocortisone was not instituted at that stage as the infant was clinically well and remained under surveillance. Increased pigmentation was noted by her parents from 6 months of age. Following a gastrointestinal illness at 8 months of age a second SST was undertaken; baseline serum cortisol was $110 \mathrm{nmol} / \mathrm{L}$ with a peak of $130 \mathrm{nmol} / \mathrm{L}$ at 60 minutes, consistent with adrenal insufficiency. Hydrocortisone therapy was commenced immediately. Fludrocortisone was not required for either child. Both siblings have normal thyroid function, $\mathrm{HbA} 1 \mathrm{c}$ and fasting glucose and insulin levels.

\section{Objective \& Methods}

To find the genetic cause of the siblings FGD whole exome sequencing was undertaken on genomic DNA of the siblings. Variants in the seven genes known to cause FGD; $M C 2 R$, MRAP, STAR, CYP11A1, NNT, MCM4 and $T X N R D 2$, were assessed for causality. Further analysis of genomic DNA and CDNA was performed by PCR/RT-PCR followed by automated Sanger sequencing.
Figure 1. (A) Pedigree of FGD family, filled symbols indicate affected individuals. (B) proband (II:2) with his unaffected sibling II:1 to his left, note his skin hyperpigmentation.
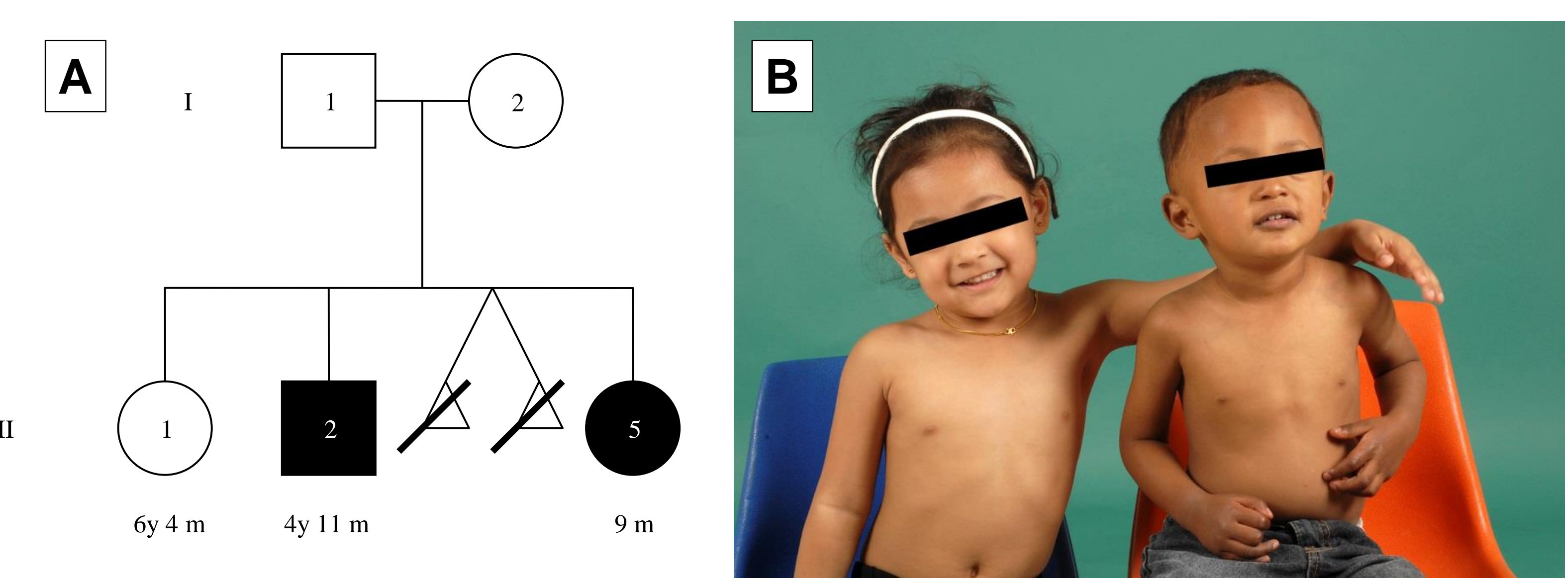

\section{Results}

Whole exome sequencing identified a novel, heterozygous variant g.5:43613069C>T; c. 211C>T; p. R71* in the antioxidant defence gene NNT, in both affected individuals II:2 and II:5 (Fig. 2A). This heterozygous R71* variant was also found in the DNA of the unaffected sibling (II:1) and their mother (I:2) (Fig. 2A). The pattern of inheritance of FGD is recessive so it was unlikely that, on its own, this heterozygous change was causative especially since it was also carried by the unaffected mother and sister. To determine whether there was an intronic lesion on the proband's other allele cDNA sequencing was undertaken after RNA protection with cycloheximide. Analysis of the patient's cDNA revealed the inclusion of a 69bp pseudoexonic sequence from intron 20 of the NNT gene [c.2995_2996insNG_032869.1:g.103679_103747] (Figure 2B \&C). If translated this would result in a frameshift and creation of a premature stop codon at position 1000, p.D999Gfs 2 . No such inclusion was seen in the cDNA of the unaffected sibling (II:1) or their mother (I:2) (data not shown). Further analysis of the genomic DNA of the patient identified a 4 bp duplication, g.[5:43701537_43701540dupAGTA], within intron 20 that is predicted by Human Splicing Finder (www.umd.be/HSF/) to create an aberrant splice acceptor site and is presumably responsible for the pseudoexon activation (Fig. 2C \&D). This change was novel, also identified in the affected sibling (II:5) and was inherited from the father (I:1) who does not have the R71* variant or FGD (2D). Neither of the heterozygous NNT sequence changes identified in this family has been annotated in dbSNP or the NHLBI exome variant server (www.ncbi.nlm.nih.gov/SNP/; http://evs.gs.washington.edu/EVS/) and both are predicted to be protein damaging.

A

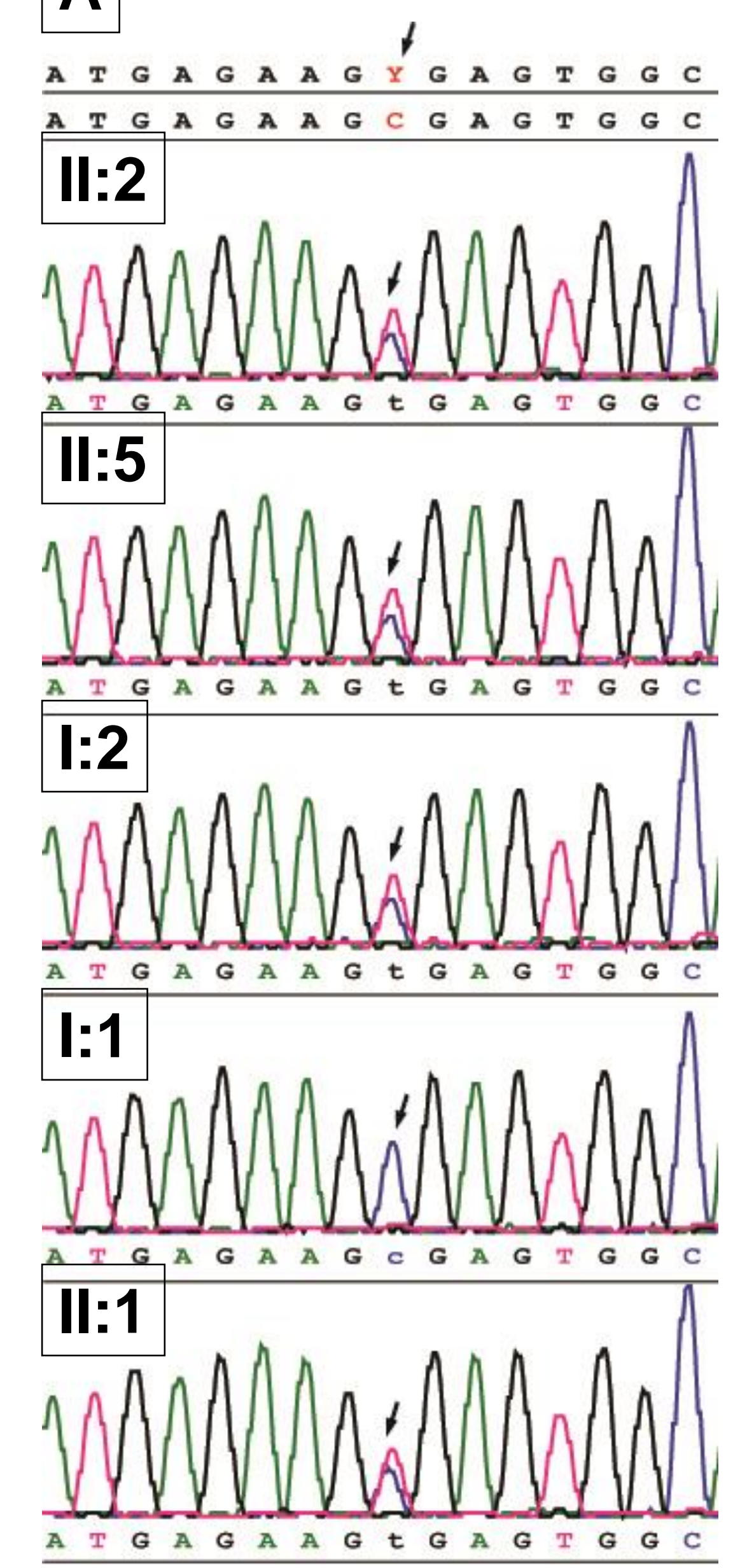

B

GTTTGGAATTCACCCAGTTGCAGGCCGAATGCCTGGTCAGCTTAATGTGCTGCTGGCTGAGGCTGGTGTGCCA TATGACATTGTGTTGGAAATGGATGAGATCAACCATGATTTTCCAGgtaagtggtgggggcaattgtgaagtttaaatattc ....tagtctccaggttctattgttgccgtatttatgttcctgagtacccagtgtttagctcccacatataagtgagaatgtgcagtatttgat ttctttcctgtgttaatttgcttaggataatggcctccagctgtttccatgttgctggaaaggacacgatttccatcctttttatggctgtgtagtatt ccatggcacatatgtaccatattttttttatccagtgcaccattgatggacatctaggttgattccatgtctttgctattgtgaatagtgctgtgatg ....agtaaagatcactataatttgtctgaaaatatgtaaaagtgaccatttgagttttatattctttctttttgttttattatatagATACTGAT TGGTCCTTGTAATTGGAGCTAATGACACTGTTAATTCAGCAGCTCAAGAAGATCCCAACTCTATTATTGCAGGC ATGCCAGTCCTTGAGGTCTGGAAATCAAAGCAG

C

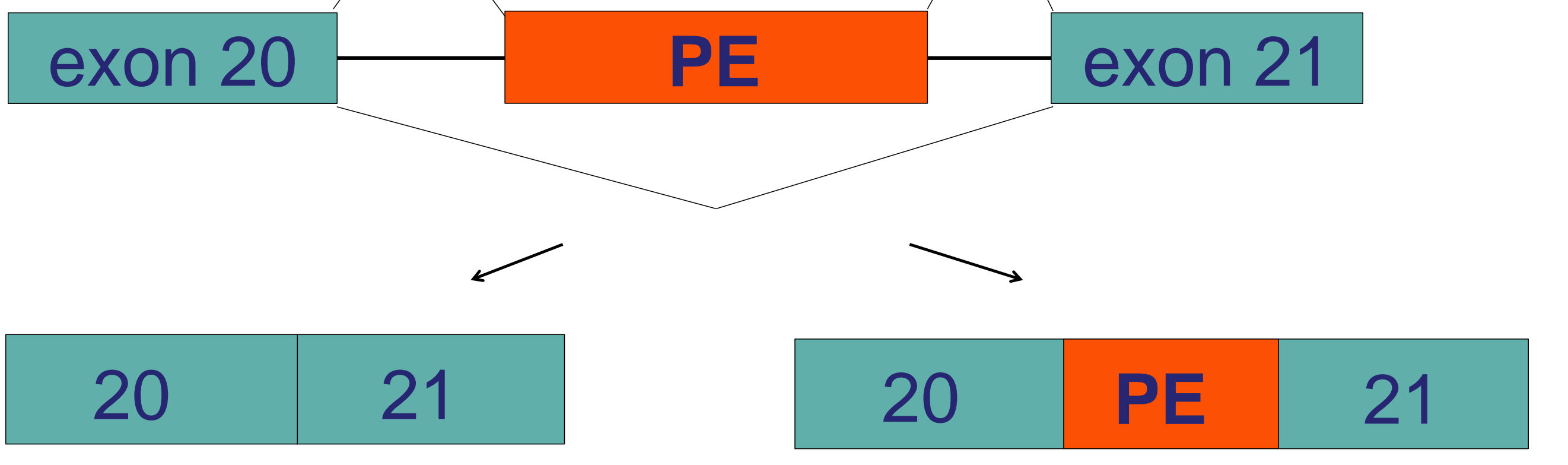

normal processing aberrant exon inclusion

\section{D}

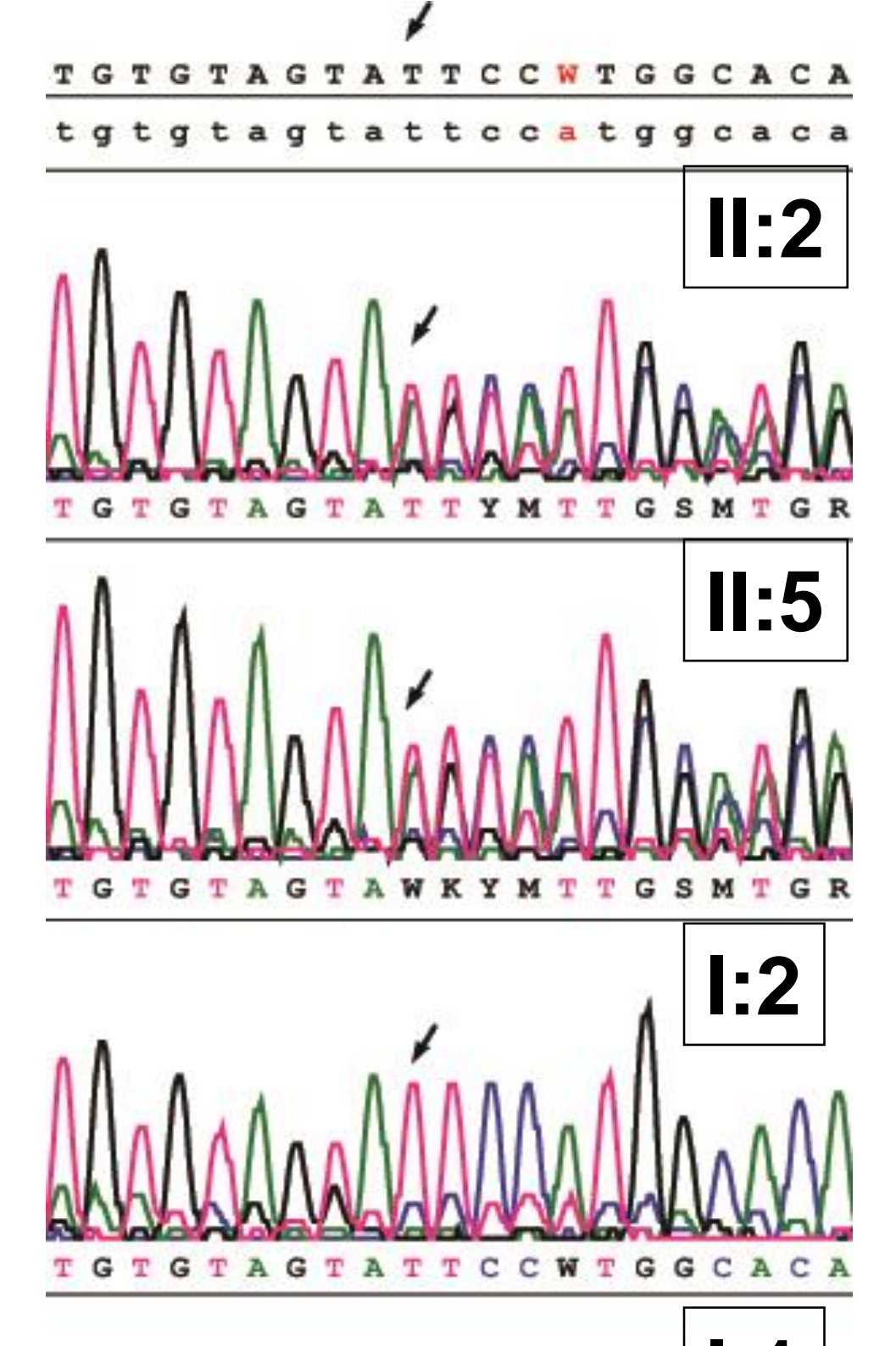

I:1
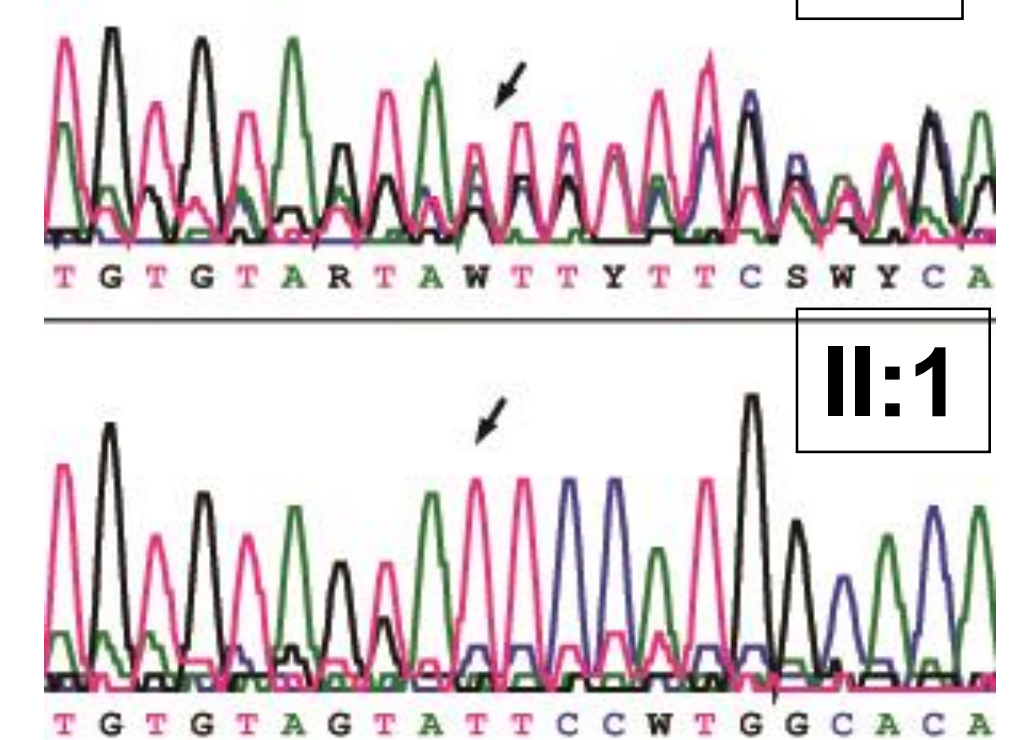

Figure 2. (A) Sequence data for the p.R71* change showing it is inherited from the father and is present in all the children (indicated with arrow). (B) The pseudoexon sequence. In uppercase are true exons 20 and 21, in lowercase is intronic sequence between exons 20 and 21. In yellow is the pseudoexon In green are splice sites and duplicated in the patient. This creates a new donor splice site and is presumably the underlying cause of pseudoexon inclusion. The red taa encodes a prematurely truncating stop codon within the pseudoexon such that its inclusion will result in a truncated protein if translated. (C) Cartoon showing the mechanism of aberrant pseudoexon inclusion. (D) Sequence data for the 4bp duplication causing pseudoexon inclusion (indicated with arrows) showing that the mutation is inherited from the mother and is present in both affected siblings but not the father nor the unaffected sister.

\section{Conclusions}

The patients reported in this study had a late onset, progressive presentation of their FGD, typical for mutations in genes involved in oxidative stress regulation. Here we report two novel, compound heterozygous mutations in NNT, including one which activates a pseudoexon, as the cause of FGD in the two siblings. Aberrant pseudoexon inclusion is rarely recognised as a cause of human disease, since, for practical reasons, intronic sequences are not usually included in mutational studies. In this case an intronic mutation was suspected because of the clue of one defective NNT allele, however, in consanguineous pedigrees, with a homozygous defect, or in dominant inheritance models this could easily be missed. This case highlights the importance of cDNA analysis to investigate the possibility of non-coding variants contributing to disease. 